\title{
Slowed Speech Input has a Differential Impact on On-line and Off-line Processing in Children's Comprehension of Pronouns
}

\author{
Tracy Love • Matthew Walenski • David Swinney
}

Published online: 3 April 2009

(C) The Author(s) 2009. This article is published with open access at Springerlink.com

\begin{abstract}
The central question underlying this study revolves around how children process co-reference relationships - such as those evidenced by pronouns (him) and reflexives (himself) — and how a slowed rate of speech input may critically affect this process. Previous studies of child language processing have demonstrated that typical language developing (TLD) children as young as 4 years of age process co-reference relations in a manner similar to adults on-line. In contrast, off-line measures of pronoun comprehension suggest a developmental delay for pronouns (relative to reflexives). The present study examines dependency relations in TLD children (ages 5-13) and investigates how a slowed rate of speech input affects the unconscious (on-line) and conscious (off-line) parsing of these constructions. For the on-line investigations (using a cross-modal picture priming paradigm), results indicate that at a normal rate of speech TLD children demonstrate adult-like syntactic reflexes. At a slowed rate of speech the typical language developing children displayed a breakdown in automatic syntactic parsing (again, similar to the pattern seen in unimpaired adults). As demonstrated in the literature, our off-line investigations (sentence/picture matching task) revealed that these children performed much better on reflexives than on pronouns at a regular speech rate. However, at the slow speech rate, performance on pronouns was substantially improved, whereas performance on reflexives was not different than at the regular speech rate. We interpret these results in light of a distinction between fast automatic processes (relied upon for on-line processing in real time) and conscious reflective processes (relied upon for off-line processing), such that slowed speech input disrupts the former, yet improves the latter.
\end{abstract}

Keywords Rate of speech $\cdot$ Pronominal resolution $\cdot$ Child language $\cdot$ Sentence processing $\cdot$ On-line and off-line processing

T. Love $(\bowtie) \cdot$ M. Walenski

School of Speech, Language and Hearing Sciences, San Diego State University, 5500 Campanile Drive, MC 1518, San Diego, CA 92182-1518, USA

e-mail: tlove@mail.sdsu.edu

T. Love $\cdot$ M. Walenski $\cdot$ D. Swinney

Department of Psychology, University of California, San Diego, La Jolla, CA, USA 
This paper explores the way in which children process sentences containing overt dependency relationships (with a focus here on pronouns) with the purpose in evaluating (a) a potential dissociation between automatic (real-time) and conscious level (off-line) processing of these constructions and (b) whether manipulating the rate of speech input has an impact on either or both of these types of processing routines.

The identification of the set of possible antecedents for a pronoun (him) is only partly dependent on the syntactic context in which it appears. Specifically, syntactic principles prohibit a pronoun from having a "local" antecedent (Principle B; Chomsky 1981, 1986; Sag and Wasow 1999). Thus in sentence 1a (see below), the pronoun (her) cannot refer to the local noun phrase (Michaela), but could refer to the non-local Alyssa (the pronoun-antecedent relationship is noted by co-indexation with the subscript ${ }_{j}$ ).

(1a) Alyssa $_{j}$ said that [Michaela threw the ball to her ${ }_{j}$ ]

(1b) Alyssa said that [Michaela $a_{i}$ threw the ball to herself $f_{i}$ ]

Note that the pronoun her in this sentence is not constrained (syntactically or otherwise) such that it must refer to Alyssa-it could refer to some other antecedent that is not explicitly mentioned in the sentence. From this potentially large set of possible antecedents (i.e., all non-local potential antecedents), the selection of the actual antecedent (i.e., determination of what the pronoun actually refers to in a given sentence) is likely to be based on semantic and/or pragmatic principles (codified as "Principle P"; Chien and Wexler 1990). In contrast, syntactic principles require a reflexive to have a local antecedent (Principle A; Chomsky 1981, 1986; Sag and Wasow 1999). This greatly restricts the set of potential antecedents, and minimizes the semantics/pragmatics in antecedent selection. Thus in sentence $1 \mathrm{~b}$, the reflexive (herself) cannot refer to Alyssa, but must refer to Michaela.

Prior research has shown that typical-language developing (TLD) children are capable of interpreting reflexives when overtly asked about the recipient of the action (Chien and Wexler 1990; Grodzinsky et al. 1993; McKee 1992). So in example (1b), children would demonstrate little difficulty (if any) correctly responding with "Michaela" when asked the question "Who caught the ball?". In contrast however, pronoun interpretation (as in sentence 1a) typically yields poorer performance in English (Chien and Wexler 1990; Grodzinsky et al. 1993; McKee 1992; van der Lely and Stollwerck 1997) and a wide range of other languages, including Hebrew, Spanish and Dutch (Grodzinsky and Kave 1994; Koster 1993; Solan and Ortiz 1982). These findings led to the suggestion that pronoun interpretation is developmentally delayed ("Principle B delay") in TLD children (Baauw and Cuetos 2003; Chien and Wexler 1990).

One potential explanation for this pattern is that TLD children demonstrate poor performance on pronouns because they have not yet mastered the semantic/pragmatic factors involved in the final selection of an appropriate antecedent (Principle P), even if they have mastered the syntactic knowledge of pronoun binding constraints (Principle B; Chien and Wexler 1990; Grimshaw 1990; Grodzinsky et al. 1993; McKee et al. 1993).

However, one commonality across these experiments is that the tasks used were off-line (i.e., temporally unconstrained) and as such require a response after the entire sentence has been processed. Examples of these techniques include act-out tasks (puppets or toys are used to act out a sentence for the child "Elmo gives him the car"- the child is then asked a question-e.g., "Who gets the car?"; Chien and Wexler 1990; Grodzinsky et al. 1993; Solan 1983), truth value judgment tasks (a sentence is presented with a picture and the participant decides YES or NO if they match; Chien and Wexler 1990; McKee 1992), and grammaticality judgment tasks (children are asked if a sentence sounds "correct"; McDaniel et al. 1990; McDaniel and Maxfield 1992). These types of post-sentential, conscious techniques 
reflect the outcomes from all levels of sentence processing including syntactic, semantic, and pragmatic processes, but cannot differentiate the contributions of these various processes to the final interpretation of the sentence, as the measurements are made after all of these processes are complete.

While these off-line techniques are quite common in evaluating pronoun comprehension, they do not allow for the moment by moment mapping of how and when such dependency relations are formed. In order to determine at what point in time a pronoun or reflexive is linked with its actual or potential antecedent(s), more temporally constrained ("on-line") techniques are needed. Such techniques allow researchers to tap in to the real time parsing of these and other sentence constructions, thus revealing distinct patterns of comprehension that off-line methods may not be able to demonstrate.

On-line studies using cross-modal priming (McKee et al. 1993; Roberts et al. 2007), selfpaced reading/listening (Booth et al. 2000), and eye-tracking (Sekerina et al. 2004) techniques have in fact evinced a different pattern of processing for pronouns and reflexives than that observed in off-line studies. In these studies, children as young as 4 years of age demonstrate on-line sensitivity to syntactic binding constraints, and show immediate linking of pronouns and reflexives to their structurally defined antecedents (for related findings in the processing of implicit anaphors, see Love 2007; Roberts et al. 2007), just as unimpaired adults do (Nicol and Swinney 1989, 2002; Nicol 1988).

These findings underscore the importance of task sensitivity. Children who demonstrate off-line difficulties in pronoun interpretation do not show deficits when measured by real time processing tasks. In the current study we will examine this tension between normal on-line pronoun processing in young children and developmentally delayed off-line performance by manipulating rate of speech input in both on-line and off-line tasks of pronoun comprehension.

As language processing depends on the timely availability of a number of types or levels of information (e.g., phonological, lexical, structural, etc.) during the course of sentence processing, rate of speech input has been implicated as an important factor in the successful processing of complex sentence constructions. When auditory information is presented to individuals (both child and adult, impaired and unimpaired) outside of the "normal" rate of 4-6 syllables/second (for English; Radeau et al. 2000; van Heuven and van Zanten 2005; Ziegler 2002), the nature of the core operations subserving structural processing may change (Camblin et al. 2007; Kail and Hall 1994; Love et al. 2008; Swinney and Love 1998; Tallal and Piercy 1973a,b, 1974, 1975; Tauroza and Allison 1990). Prior research suggests that these changes may be disruptive in some cases but beneficial in others.

For unimpaired adult listeners, slowed input rates appear to impair the automatic processing routines underlying the establishment of structural dependency relationships during real time language comprehension in real time tasks (Love et al. 2008). Off-line studies with these unimpaired populations demonstrated a similar decline in performance (Swinney and Love 1998). In contrast, reports suggest that neurologically compromised individuals-e.g., adults with Broca's aphasia or language impaired children-may benefit from a slowed rate of input for both on- and off-line tasks (Gardner et al. 1975; Lasky et al. 1976; Love et al. 2008; Pashek and Brookshire 1982; Poeck and Pietron 1981; Tallal and Piercy 1973a,b, 1974, 1975). Interestingly, Broca's patients evinced deficits in parsing syntactic dependencies (in object-relative clauses, like "The audience liked the wrestler that the parish priest condemned for foul language.") when sentences were presented at "normal" rates, and showed improved performance and more normal-like parsing routines with slowed speech input (Love et al. 2008). 
In the current cross modal priming study we will investigate the impact of a slowed rate of speech input on the comprehension of pronouns and reflexives in typical-language developing children in temporally sensitive on-line tasks and temporally unconstrained off-line tasks. ${ }^{1}$ Based on prior results (see above), at a regular rate of speech input we expect to find immediate linking of a pronoun with its antecedent (i.e., adult-like performance) on-line, but impaired performance off-line, consistent with a developmental delay for pronouns. At a slowed rate of speech input, performance on pronouns on both on-line and off-line tasks may be impaired, as previously found for syntactic processing with slowedspeech input in unimpaired adults. Alternatively, on-line performance may be unaffected at slowed input rates, whereas off-line performance may be improved as children may not have explicitly mastered these co-reference constructions, and may therefore benefit from slowed input, as previously found for impaired adults. A third possibility is that children may show impairments with slowed input for those aspects of co-reference processing that they have mastered (e.g., on-line determination of the antecedent of a pronoun), but show improvements at aspects of processing that they have not yet mastered (e.g., off-line interpretation of pronouns).

\section{Experiment 1: On-line Co-reference Processing and Rate of Speech}

In this experiment we investigate the real-time nature of pronoun-antecedent linking in children (ages 5-13) at both regular and slowed rates of speech input. To investigate these processes as they unfold in real time, we will use a variant of the cross-modal priming (CMP) technique (Swinney 1979) — cross-modal picture priming (CMPP) — that has been used previously in the investigation of co-reference processing in children (Love 2007; McKee et al. 1993; Roberts et al. 2007; Swinney and Prather 1989). Cross-modal priming itself has been used extensively in the adult syntactic processing literature to assess, among other things, how and when a reference-seeking element (e.g., pronoun, reflexive) is linked to an antecedent (for review, see Nicol and Swinney 2002).

In Cross Modal Lexical Priming (CMLP; Swinney et al. 1979) participants listen to auditorily presented sentences and simultaneously make a binary decision (typically a word/nonword lexical decision) to letter strings presented on a screen in front of them. An element in the on-going auditory sentence (e.g., a word or a pronoun) serves as a prime for the letter strings (target), which are either semantically related (related target) to the sentence-prime or serve as a semantically unrelated baseline (control target). Priming effects are found when the response times to the related targets are faster than response times to the control targets-but crucially only when they are presented immediately (or very soon) after the prime in the sentence. Related and control targets are typically matched, such that their response times don't differ in other sentence positions (not near the prime) or when presented in isolation (e.g., in a visual lexical decision task).

Of importance with this technique is that fact that (a) the presentation of the auditory sentence is never ended with the presentation of the visual target, thus mitigating the possibility of integration of the visual stimulus into the ongoing auditory presentation of the sentence,

\footnotetext{
1 We note that CMLP has been shown to be insensitive to integration effects when the auditory stimuli is presented within a normal speech rate of 4-6 syllables/second (for English; Radeau et al. 2000; van Heuven and van Zanten 2005; Ziegler 2002). At slowed presentation rates ( $<4$ syllables/second), visually presented words may be integrated into the ongoing auditory speech stream when the visual probe constitutes a good continuation of the sentence ( Nicol et al. 2006). In this study, our visual probes are pictures rather than words, and thus we believe that these visual stimuli do not lend themselves to such integration effects.
} 
(b) the presentation of the auditory sentence continues past the presentation of the visual target so that the process revealed is not obscured by end-of-sentence wrap-up effects and (c) participants never make conscious, deliberate, meta-linguistic judgments about the sentences that they hear (e.g., Is it grammatical? Did I just hear a /p/ sound?). In other words, the judgments participants make are based solely on consideration of the visual targets; participants need not consciously evaluate each successive word of the auditory sentence. (For further details, see: Balogh et al. 1998; Love and Swinney 1996; Nicol et al. 1994, 2006).

A variant of this technique that has been successfully used with child and adult populations - both language unimpaired and language impaired, uses pictures in place of letter strings as the related and control targets. This Cross-Modal Picture Priming (CMPP) paradigm (Swinney and Prather 1989) is particularly useful for children and language-impaired adult participants, for whom words or non-word letter strings may be difficult. Rather than a word/non-word decision, participants make simple classification decisions to the pictures (e.g., 'Can I eat it?,' 'Is it alive or not?'). Importantly, the time to make these classification decisions to pictures has been demonstrated to be speeded (primed) if the picture bears a relationship to a word just heard in a sentence, for both children and adults (Love 2007; McKee et al. 1993; Roberts et al. 2007; Swinney and Prather 1989; Swinney et al. 1996).

In the current study, we use a matched sentence (as opposed to matched target) paradigm (for details of this variation with CMLP, see Cowles et al. 2007; Nicol et al. 1994; Walenski 2002). Here, rather than presenting related and control pictures for each sentence, the same picture is presented to two (minimally) different sentences - one of which contains a prime and one of which doesn't (McKee et al. 1993). Since the same picture is used in the primed and unprimed conditions, this eliminates the concern that differences in response time indicative of priming could be due to differences between two different pictures. See just below for further details.

\section{Method}

\section{Participants}

Forty-three native English speaking children ages 5-13 participated in this study (mean age $=7.43$ years, $\mathrm{SD}=1.80$ ). Twenty-two participants were boys, 21 girls. All participants met the following criteria: absence of frank neurological impairment, social/emotional disorders (e.g., autism), and absence of hearing (via GSI-17 audiometer screening pure tone sweep from 250 to $8,000 \mathrm{~Hz}$ at $20 \mathrm{~dB}$ ) or vision impairments. In addition, all participants were within or above the average range ( 85 or above; mean $=113.67, \mathrm{SD}=12.10$ ) on the Test of Non-Verbal Intelligence-3 (TONI-3; Brown et al. 1997), and were well within the normal range (core language index of 95 or above; mean $=115.26, \mathrm{SD}=10.10$ ) on the Clinical Evaluation of Language Fundamentals-4 (CELF-4; Semel et al. 2003). Participants for this study were recruited from schools in the San Diego Unified School District and Del Mar School District. After the language assessment was completed, parents brought the children to our laboratory for multiple visits, each lasting approximately 1 hour (Experiment 1: 3 visits; Experiment 2: 1 visit). After each visit, parents of participants were compensated $\$ 5$ for traveling expenses, and the children were allowed to choose a prize. Upon completion of the study, each participant was given an additional \$25. 
Design and Materials

In this Cross-Modal Picture Priming (CMPP; Love 2007; Roberts et al. 2007; Swinney and Prather 1989) task, children listened to sentences over headphones. At specific moments in the sentence, a picture would appear on the screen. Children were instructed to listen carefully and try to understand the sentences as they would be questioned about them from time to time. The children were also told they would have to make a button-press decision to the visual targets, deciding as quickly as possible whether or not the item pictured was "alive" (right button response) or "not alive" (left button response).

The on-line stimuli for this experiment were taken from a similar study by McKee et al. (1993). The thirty experimental sentences had the bi-clausal structure illustrated in (2; below), with the complement clause containing either a pronoun (him) that is co-referential with the main clause subject (e.g., bird; 2a); a reflexive (himself) that is co-referential with the complement clause subject (e.g., turtle; 2b); or a non-co-referential noun phrase (e.g., the lady; 2c) that serves as a control condition. Note that as response time comparisons are made to the same picture across sentences in this task (i.e., there is no within-sentence baseline, as there typically is in other cross-modal priming experiments; see above), differences in processing load for different sentences are a potential concern. It has been argued that the additional semantic referent (lady) in the control condition may create a higher processing load than the sentences which do not have this additional referent-which may therefore lead to the appearance of priming in the pronoun condition, due to elevated response times in the control condition (Hestvik et al. 2007). However, the reflexive condition can serve as another point of comparison, as it does not introduce a new referent, and may therefore be a close match to the pronoun-containing sentences with respect to processing load. Even this is not a perfect match however, as reflexives are restricted to local antecedents only, and therefore may actually engender less of a processing load than pronouns, leading to faster response times in the reflexive condition. This load difference therefore works against a prediction of a priming effect (faster response times) in the pronoun condition, and so the observation of such a priming effect would mitigate this potential concern.

As we are interested in investigating pronoun co-reference, a picture corresponding to the main clause subject (e.g., bird) was presented at the offset of the pronoun, reflexive, or noncoreferential noun phrase (all experimental items required an "alive" answer) for all versions of the experimental sentences. Therefore, the picture was consistent with the correct antecedent for the pronoun (2a), but not the reflexive (2b) (see Fig. 1 for an example). Note that this is different from the McKee et al. (1993) study, for which the picture corresponded to the correct antecedent for the reflexive. The sentences were constructed so that there were at least seven syllables after the point that the picture was presented in order to ensure that participants would continue processing auditory sentence material during the decision task. For the pronouns and reflexives, the third-person singular masculine form was used for all sentences. For the non-coreferential noun phrases 10 different female non-animal nouns were used.

(2a) Pronoun: The bird says that the turtle with the hard shell is rubbing him [BIRD] with suntan oil on the sandy beach.

(2b) Reflexive: The bird says that the turtle with the hard shell is rubbing himself [BIRD] with suntan oil on the sandy beach.

(2c) Control: The bird says that the turtle with the hard shell is rubbing the lady [BIRD] with suntan oil on the sandy beach.

In addition to the experimental items, 30 filler sentences were created that were similar in length and structure to the experimental sentences, and either contained a pronoun or reflexive 


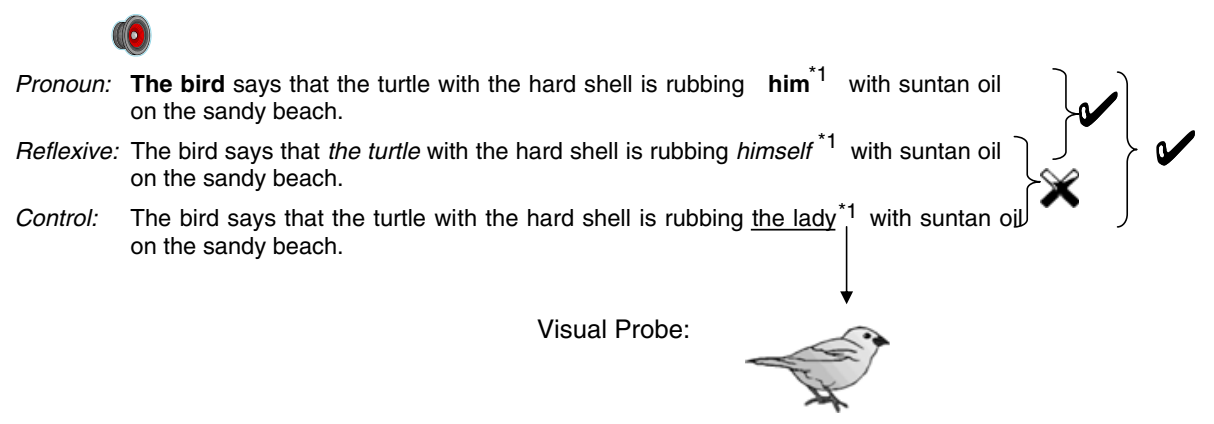

Fig. 1 Examples of matched sentence (pronoun, reflexive, unrelated NP) conditions and visual target (bird), with predicted patterns of priming (pronoun vs. control; reflexive vs. control) at a regular rate of speech input. Note that a check mark indicates a prediction of priming for the antecedent of the pronoun and the x-mark indicates no predicted priming in the reflexive condition. See text for predictions for the slowed speech rate conditions

( $n=10$; see 3a below) or did not ( $n=20$; see $3 \mathrm{~b}$ below). For the filler sentences, pictures of inanimate objects (requiring a "not alive" answer) were presented at various points in the sentence. The filler sentences (which were the same across all scripts) were constructed to reduce the expectation of an "alive" response following a pronoun or reflexive (as several of the fillers contained a pronoun or reflexive), and to balance the number of "alive" and "not alive" responses.

(3a) The camel thinks that the ostrich jumping over the bed will hurt him [BED] and everyone will have to stop playing

(3b) The alligator under the umbrella wonders if the fox in [UMBRELLA] the river is taking a bath this afternoon.

In this mixed factorial design, the experimental sentences were counterbalanced across three scripts, such that each script contained only one version of any particular sentence (pronoun, reflexive, control), but contained an equal number of exemplars of each condition (10; note that the same non-co-referential noun phrases were used in all three scripts, but did not repeat in any single script). The filler sentences were pseudo-randomly intermixed into the script with the experimental sentences, such that no response type (alive, not-alive) occurred more than three times in a row. The first six items in each list were filler items, intended as practice (participants were not told this), to ensure that the children were in the rhythm of the task before the experimental items started. Every participant received all three scripts (i.e., this was a within-subjects design) though each script was presented on a separate (1-hour) visit, with at least 3 weeks between visits, to minimize repetition effects. Thus only one version of each sentence appeared on a given visit and each picture was also only seen once per visit. Each child returned three times to complete the experiment.

In addition, we also manipulated the rate of auditory speech input-presenting the sentences at both a regular and a slowed rate of input (in separate experiments). Sentences were recorded by a female native speaker of English at a regular speech rate ( $m e a n=5.03$ syllables per second). The regular rate sentences were then digitally slowed (using the Cool Edit Pro v1.2 software package) to $2 / 3$ of the regular rate (mean $=3.36$ syllables per second). Rate of speech input was run as a between-subjects factor, with different subjects hearing the sentences at each rate (regular, slow). Participants reported that the slow rate sentences sounded "OK....[but that the] speaker sounded tired." 
Procedure

Prior to testing, the participants went through extensive training and practice to ensure both that they understood the dual nature of the task (listen to and comprehend the ongoing auditory sentences; make a button-press decision to the pictures), and that they could perform both tasks simultaneously. The training proceeded in stages. In stage one, the experimenter held a set of half-sheet $(8.5 \times 5.5 \mathrm{inch})$ training cards with training pictures printed on them (7 alive/7 not alive). The children were taught to verbally respond "yes" or "no" with regard to the alive-ness of the depicted object. If a participant mis-categorized an item, the experimenter would talk through the item until correctly identified. In stage two, the children were introduced to a button box, where the right button indicated a "yes" (alive) response and the left button indicated a "no" (not alive) response. The experimenter used the same set of training cards from stage one, but this time the children responded (non-verbally) by pressing the buttons on the button box. If the participant demonstrated difficulties during this training section, the experimenter gave direct feedback similar to that in stage one. In stage three a training script was used whereby the pictures were presented on the computer screen. The children were instructed to watch the computer screen and make a binary alive/not-alive decision about the pictures using the button box as quickly as possible. This script was broken down into four blocks of five pictures each. In the first block, the picture remained on screen for $1.5 \mathrm{~s}$; in the remaining blocks, the picture remained on screen for only $0.5 \mathrm{~s}$ (this reduction in picture presentation time over the course of training was to encourage participants to respond to the pictures in a speeded fashion, while maintaining high accuracy). Once again, if the children had any difficulty with this, feedback was given during the pauses between segments. This training script did not include sound.

Finally, stage four introduced the children to the cross modal experimental paradigm. The children were told that they would be listening to sentences while watching the computer screen for pictures at the same time. At specific moments in the sentence, a picture would appear on the screen. The children would have to decide as quickly as possible whether or not the item pictured was "alive" or "not alive." The children made their decisions by pressing one of two large red buttons on a button box that they could rest their arms and hands on. The right button was used for a YES response (it is alive: picture of a koala) whereas the left button was used for a NO response (it is not alive: picture of a cupcake). During this section, participants are trained to $100 \%$ button press response criterion and are also given comprehension questions to ensure they understand the practice sentences.

Stimuli were presented with an in-house software program (TEMPO; version 2.1.2) to control the presentation of the pictures and sentences, and collect responses. A millisecondaccurate computer timer was initiated with onset of the presentation of the picture. The timer terminated with the participant's button press, recording both the response and the time to respond. Each picture remained on the screen for $1,500 \mathrm{~ms}$ or until a button press was made (whichever came first), and responses were allowed for an additional 1,000 ms, leaving a $2,500 \mathrm{~ms}$ window within which a participant could respond. Any response times longer than $2,500 \mathrm{~ms}$ were recorded as a NO RESPONSE and counted as an error. There was a three second gap between successive sentences.

To encourage attention to the sentences, the experiment was periodically paused and the participants were asked a multiple-choice question ( 3 answer choices per question) about the sentence that had just been presented (18 questions per session, three of which were paired with filler sentences). The questions were distributed approximately evenly over the course of the script, and targeted the participants' knowledge of the actors in the sentence or the location in which the sentence occurred. The questions that were asked were intended 
only to reinforce the need for the subjects to listen to the sentences, rather than as a test of their anaphor comprehension per se. The questions for script 2 were different from those of scripts 1 and 3, which were the same. This allowed us to evaluate practice effects on the comprehension questions across scripts. No such practice effects were found for either speed of presentation. After the first visit, the training cards were no longer used, but the training script without sound was used to re-familiarize the children with the task. Each experimental script took about $20 \mathrm{~min}$ to complete.

Analysis

Prior to analysis, data from one subject were removed from the regular speech rate condition due to chance accuracy (51\% correct-which was also more than four standard deviations lower than the mean accuracy of all subjects) on the button press judgment task. In addition, two subjects in the regular speech rate condition did not complete all visits for this experiment, and were excluded.

Prior to inferential statistics, the data from the remaining 40 participants (18 at regular rate, 22 at slow rate) were screened (removing $1.1 \%$ of all data) to help reduce variability and avoid outlier response times: all non-responses and incorrect responses were excluded (note that response times less than $300 \mathrm{~ms}$ were excluded as errors). $z$-scores were computed for each participant's correct responses for each visit, and any score greater than 3 or less than -3 was excluded; $z$-scores were then computed for each item (picture) at each rate of speech, and any score greater than 3 or less than -3 was excluded. As a final check, after removing both incorrect responses and response time outliers, we checked the number of remaining data points for each subject and each item. While no subjects stood out from the rest in this respect, three items were noteworthy in having few data points remaining. For two of the items (bird, squirrel), it was discovered that the audio recordings had become corrupted, likely contributing to the high error and outlier rates on these items. The third item (alligator), had the highest rate of excluded data of any of the items (20\% of data points were excluded), though no obvious reason for this high error rate was apparent. As all three of these items appeared to be yielding abnormal response patterns, all data from these three items were excluded. This careful screening procedure was necessary to ensure that any response time differences between conditions would not be overshadowed by the high subject- or item-based variability inherent to response times from young children on this task.

The remaining data from 27 items (screened correct first responses) were ln-transformed to reduce skewness and kurtosis in the distribution of response times. Data were analyzed using a mixed-effects regression model, with crossed random effects of subject and sentence (which combine traditionally separate F1 and F2 analyses in a single statistical test), and fixed effects of speech rate (regular, slow) and sentence condition (pronoun, reflexive, control). In addition, variables coding item-order, visit number, and their interaction were included as covariates to control for variability introduced by any change in performance as the task progressed (order), differences in performance across sessions (visit), or differences in the change in performance across the task for different visits (order $\times$ visit interaction). These three covariates were also included as random-effects factors (accounting for individual differences in these effects). Subsequently, follow-up planned comparisons were made within each rate (i.e., within-subjects) for control-pronoun; pronoun-reflexive; and control—reflexive differences in response time (i.e., follow-up comparisons were not made for between-subjects differences). 
$F$-statistics are reported for main effects and interactions, and $t$-statistics for planned comparisons of sentence type differences (within each rate). All $p$-values from $t$-statistics are reported two-tailed, unless otherwise noted. In all analyses, degrees of freedom were computed using the Satterthwaite approximation (Satterthwaite 1946). Note that the degrees of freedom are large because in these regression models they are based on the number of data points, not the number of subjects or items. Note also that degrees of freedom may be fractional as they are computed based on variance estimates. For similar analyses, see Walenski et al. (2007), and Love et al. (2008) and for further discussion of these methods of analysis, see Baayen (2004, 2008).

Results for Regular and Slowed Rates of Speech Input

Accuracy at the button press decisions was quite good ( $91.5 \%$ over all conditions), and about the same across all three conditions at both regular (pronoun: $91.8 \%$; reflexive: $92.8 \%$; control: $92.2 \%$ ) and slowed (pronoun: $90.6 \%$; reflexive: $92.9 \%$; control: $88.7 \%$ ) rates of speech input. The comprehension questions were answered at $82 \%$ accuracy (range: 62-98\%) at the regular speech rate, and at $84 \%$ accuracy (range: 64-100\%) at the slowed-speech rate, suggesting that the children were attending to and understanding the auditory input.

For the response time data (Fig. 2), the children responded comparably fast at regular and slowed rates of input (main effect of rate: $F(1,38)<1$ ), though the interaction between input rate and condition (sentence type) was significant $(F(2,2778)=3.86, p=0.021)$.

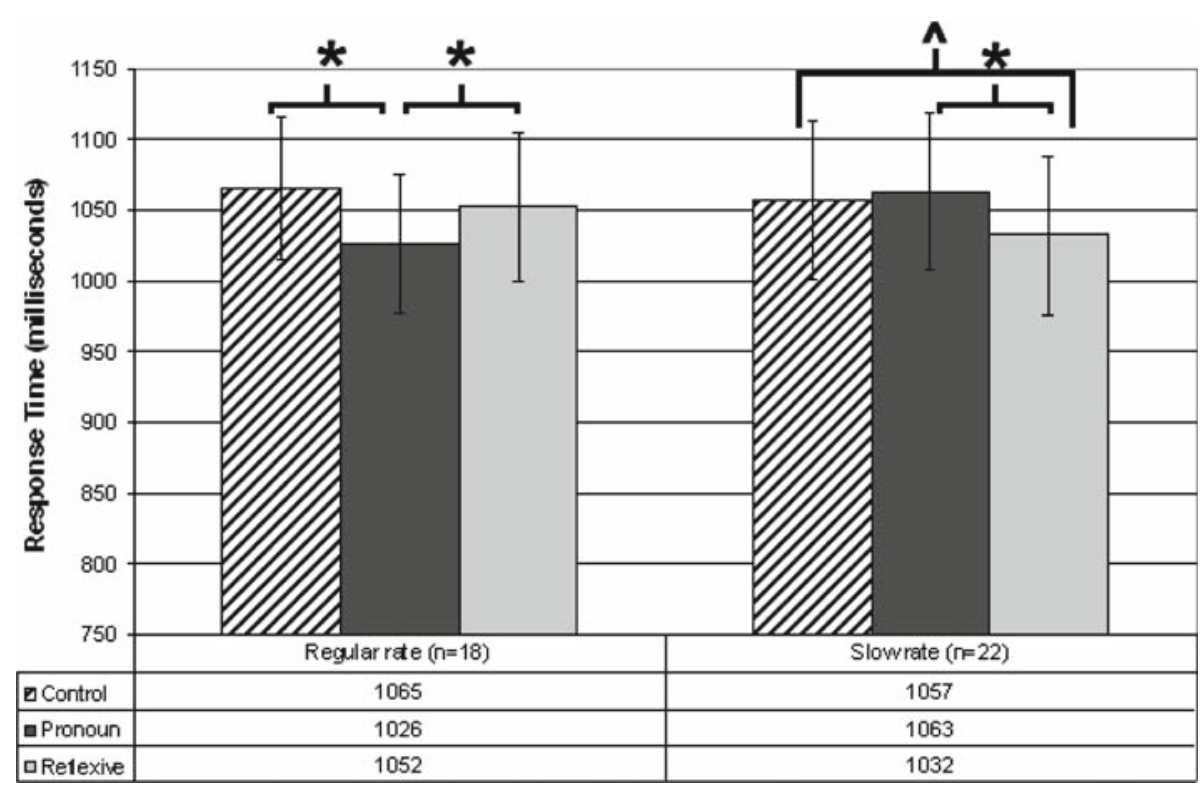

"The bird ${ }_{\mathrm{i}}$ says that the turtle $\mathrm{j}_{\mathrm{j}}$ with the hard shell is rubbing $\mathbf{h i m}_{\mathrm{i}} /$ himself $_{\mathrm{j}}$ the lady $_{\mathrm{k}}{ }^{{ }_{1}}$ with suntan oil on the sandy beach."

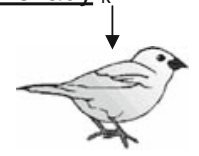

Fig. 2 Response times (error bars indicate standard error) at regular and slowed rates of speech input. Star ('*') indicates a significant comparison $(p<0.05)$; caret ( $\left.{ }^{\text {‘’ }}\right)$ indicates trend significance $(p<0.10)$ 
At a regular rate of speech input, children were reliably faster at the probes in the pronoun condition (mean response time $=1,026 \mathrm{~ms}$ ) than in the control condition (mean response time $=1,065 \mathrm{~ms}$ ), consistent with priming for the correct antecedent of the pronoun $(t(2779)=2.33, p=0.020)$. In contrast, the difference in response time between the control and reflexive sentences did not even approach significance $(t(2776)<1)$, consistent with no priming for the incorrect antecedent of the reflexive. In other words, at regular rates of speech input, antecedent assignment appears to be functioning normally for young children (age 5-13), for both pronouns and reflexives, as expected. In addition, to verify that the pronoun priming effect was not due to differences in processing load between the control and pronoun sentences, we compared the pronoun and reflexive conditions. The response times in the pronoun condition were faster than those in the reflexive (incorrect antecedent) condition (mean response time $=1,052 \mathrm{~ms}$ ), a difference that approached significance $(t(2779)=1.77, p=0.077$, two tailed), suggesting that the priming effect for pronouns was not an artifact of an extra processing load in the control condition.

At slowed rates of speech input, however, the pattern changes. Here the fastest response times were in the reflexive condition (mean response time $=1,032 \mathrm{~ms}$ ). The difference in response time between the reflexive and control condition approached significance, as measured via a two tailed t test (control mean response time $1,057 \mathrm{~ms} ; t(2777)=1.81, p=$ 0.070 ), and the response time difference between the reflexive condition and the pronoun condition reached significance (pronoun mean response time $=1063 \mathrm{~ms} ; t(2777)=2.08, p=$ 0.038 , two tailed). There was no difference in response time for the control and pronoun sentences $(t(2778)<1)$. These results suggest incorrect assignment of the antecedents with slowed-speech input, for both the pronouns (which failed to prime the correct antecedent) and reflexives (which primed the wrong antecedent).

\section{Discussion}

At a regular rate of speech, TLD children exhibit faster response times to pictures consistent with the antecedent of the pronoun only when those pictures are presented at the offset of the pronoun, but not when they are presented at the offset of a reflexive or an unrelated noun phrase. This is consistent with correct co-reference processing of pronouns in typically-developing children.

At a slowed rate of speech input, TLD children show an abnormal pattern of priming, with faster response times in the reflexive condition than the pronoun or unrelated noun phrase condition. This suggests that the antecedent of the pronoun is not being correctly assigned, as no priming is found following the pronoun condition for a picture of its antecedent. Moreover, it also suggests that the antecedent of the reflexive is not being assigned normally, as a syntactically unlicensed antecedent is being primed following the reflexive. Note however that we did not examine in this study whether or not the syntactically appropriate antecedent of the reflexive may also be primed, so this result could be consistent with either aberrant priming (only the wrong antecedent is primed), or excessive priming (multiple noun phrases are primed as potential antecedents). In either case, on-line syntactic processing appears to break down with slowed input.

Finally, as we included children over a wide range of ages, we wanted to ensure that the priming effects (particularly for the regular speech rate) were not being primarily driven by (e.g.) just the older children. To address this possibility, we re-ran the regression model with age (as a continuous variable; specified in years) and its interactions with rate and condition included. There was a significant main effect of age (older children responded more 
quickly; $F(1,36)=4.68, p=0.04)$, but neither of the interactions reached significance (all ps $>0.60$ ), consistent with similar priming effects in children across the range of ages tested-that is, children even as young as 5 years old don't appear to be different than the older children, and appear to be priming the antecedent of the pronoun normally, consistent with prior studies of on-line co-reference processing in young children (Love 2007; Roberts et al. 2007).

\section{Experiment 2: Off-line Comprehension of Pronouns and Reflexives at Different Speech Rates}

This experiment investigates the off-line comprehension of explicit anaphors (pronouns and reflexives) at regular and slowed rates of speech input, using an off-line sentence/picture matching task.

We expect that at regular rates of speech typical-language developing children will perform worse on the interpretation of pronouns than on the interpretation of reflexives, consistent with prior claims of a developmental delay for pronouns (see above). At slowed rates of speech input, one possibility is that performance on pronouns may be improved relative to performance at regular rates of speech input, as previously found for language-impaired adults (see above). A second possibility is that performance on pronouns will be worse with slowed input (relative to the regular input rate), as previously found for unimpaired adults (see above), though this would perhaps be inconsistent with a developmental delay for pronouns (which in this sense is perhaps analogous to a language impairment on these forms, albeit one that the children grow out of). As there has been no such observation of a developmental delay for reflexives, we do not expect performance on reflexives to improve with slowed input, but may be the same or possibly worse (as automatic processing routines are disrupted by the slowed input).

\section{Method \\ Participants}

The same group of children was used for this Experiment as for Experiment 1. All children completed this experiment at the same rate at which they received the on-line materials.

\section{Design and Materials}

We chose 15 of the sentences from experiment one that were highly imageable, and hence could be represented as pictures for our sentence/picture matching task (this number of items offered a compromise between a sufficient number for reliable results, and not so many that this untimed task would take the children too long to complete). These fifteen sentences were modified for this experiment by removing the first noun phrase from the on-line experimental sentences used in Experiment 1 (repeated here as 4a) and creating stand-alone sentences out of the relative clauses. There were two versions of each sentence, one with a pronoun (4b), and one with a reflexive (4c) resulting in a total of 30 stimuli. Each participant was given all 30 stimuli in the same session, resulting in repetition of items that differed only with respect to whether the sentence contained him or himself. Rate was manipulated as a between-subjects 
factor. For each rate, the experimental sentences (pronoun, reflexive) were all presented in a single list, with the two versions of a given sentence (pronoun, reflexive) as far apart in the list as possible.

(4a) The bird says that the turtle with the hard shell is rubbing himself with suntan oil on the sandy beach.

(4b) The turtle with the hard shell is rubbing him with suntan oil on the sandy beach.

(4c) The turtle with the hard shell is rubbing himself with suntan oil on the sandy beach.

The regular rate sentences were recorded at an average rate of 5.00 syllables per second by the same female native English speaker. These sentences were digitally slowed using the same methods described in Experiment 1, rendering sentences with an average rate of 3.19 syllables per second. Each sentence was paired with an $8.5 \times 11$ inch picture card with two picture scenes placed one above the other. One scene was consistent with the meaning of the pronoun version of the sentence, while the other depicted the reflexive version of the sentence (Fig 3).

\section{Picture Pre-tests}

Once the materials were constructed, they were pretested to ensure that the sentence containing the reflexive would match the reflexive-interpretation picture and not the pronoun-inter-

"The turtle ${ }_{\mathrm{j}}$ with the hard shell is rubbing him $_{\mathrm{i}} /$ himself $_{\mathrm{j}}$ with suntan oil on the sandy beach."
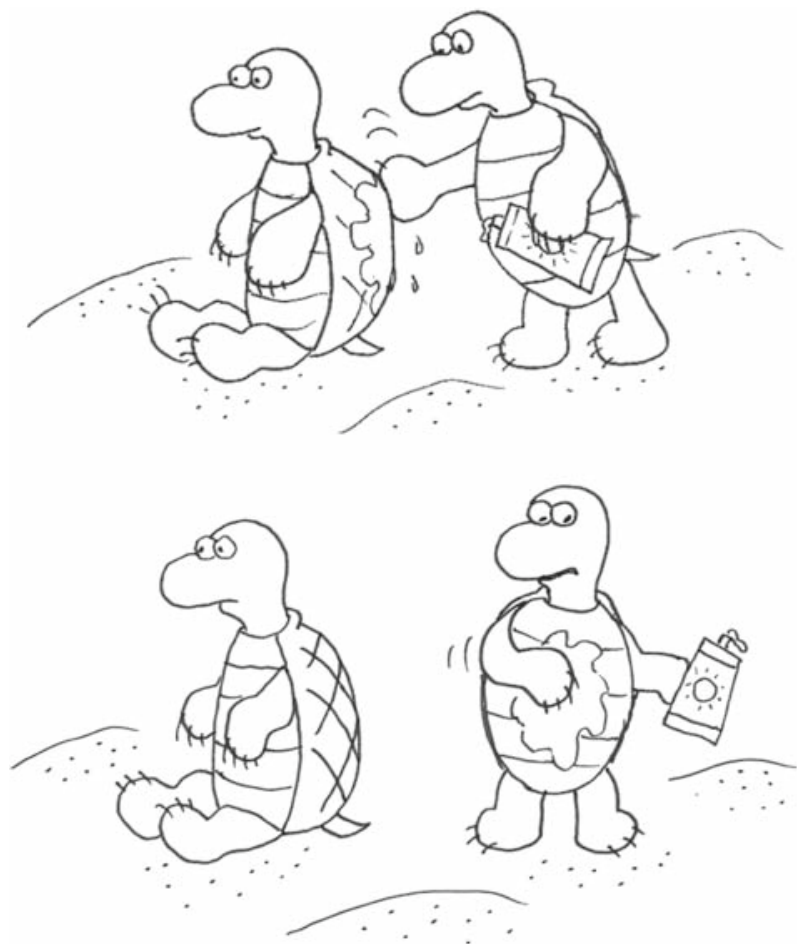

Fig. 3 Example of a sentence and corresponding picture card for the off-line sentence/picture matching task 
pretation picture; and, conversely, that the sentence containing the pronoun would match the pronoun-interpretation picture and not the reflexive-interpretation picture. Twenty-two native English speaking UCSD undergraduates (mean age $=20.32 ; \mathrm{SD}=1.04$; all were women) rated the relatedness (from 1 to 7; 1 indicated "no relation", 7 indicated "highly related") of each sentence for its correct and incorrect picture. For the matching items (reflexives with the reflexive picture, pronouns with the pronoun picture), the mean relatedness score (averaged across subjects) was 6.31 ( $\mathrm{SD}=0.56$; range: $5.05-7.00)$. For the mismatching pictures (reflexives with the pronoun picture, pronouns with the reflexive picture), the mean relatedness score was 1.53 ( $\mathrm{SD}=0.49$; range $1.05-2.91)$. This difference was statistically reliable (unpaired $t$-test on item means: $t(58)=35.2, p<0.0001$ ) indicating a single, clear correct choice between the two pictures.

\section{Procedure}

The off-line experiment was conducted on a separate visit scheduled at least a week after their final visit for the on-line portion of the study (Experiment 1). For this task, the children were instructed to listen carefully to the sentences and to point to the one picture out of the two choices that best matched the sentence that they just heard. The participants were familiarized with the task with four practice items (read to them by the experimenter) prior to the presentation of the experimental stimuli. The sentences were played on a CD player, and the picture cards were placed in a stack on the table. The experimenter turned to the next picture card after each sentence. The experiment took about 20 min to complete.

\section{Analysis}

Data were analyzed using a mixed-effects logistic regression model for binary data, with crossed random effects of subject and sentence, and fixed effects of speech rate (regular, slow) and sentence condition (pronoun, reflexive). Follow-up comparisons were made within each sentence type (pronoun, reflexive) contrasting regular and slowed input.

$F$-statistics are reported for main effects and interactions, and $t$-statistics for follow-up comparisons. All $p$-values from $t$-statistics are reported two-tailed, unless otherwise noted. In all analyses, degrees of freedom were computed using the Satterthwaite approximation (Satterthwaite 1946). Again, note that the degrees of freedom are large because they are based on the number of data points in these regression models, not the number of subjects or items. For similar logistic analyses, see Walenski et al. (2007). For further discussion of these methods of analysis, see Baayen $(2004,2008)$.

\section{Results at Regular and Slowed Input Rates}

For this task (Fig. 4), performance on reflexives was better than on pronouns at both input rates (main effect of sentence-type: $F(1,28.83)=58.85, p<0.0001$ ), and the interaction between input rate and sentence-type was significant $(F(2,1286)=30.55, p<0.0001)$. The main effect of input rate was not significant: $F(1,33.27)=1.42, p=0.242$. The significant interaction results from a large improvement in accuracy on the pronoun sentences with slowed input relative to the regular rate of speech input (from $37 \%$ to 66\%, an improvement of $29 \%$; $t(36.52)=3.13, p=0.003)$, with no improvement in accuracy for 


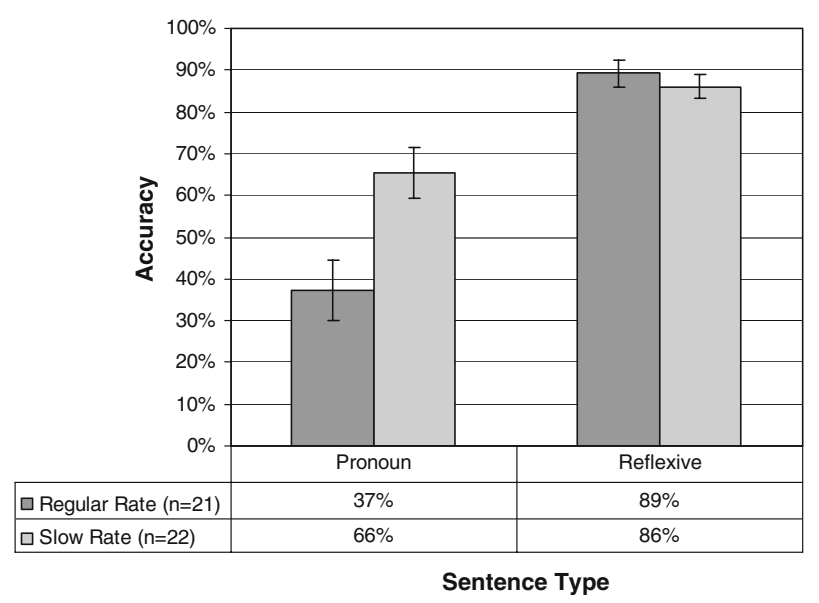

Fig. 4 Mean accuracy (percent correct; error bars indicate standard error) in the off-line task for pronouns and reflexives and regular and slowed rates of speech input

the reflexive sentences $(89 \%$ at a regular input rate vs. $86 \%$ at a slowed input rate; $t(48.9)=$ $0.74, p=0.463)$. We note that the mean accuracy score of $37 \%$ for the pronoun condition with regular rate input was marginally worse than chance $(50 \% ; t(20)=1.79, p=0.088)$. This poor performance improved to a reliably-better-than-chance $66 \%$ with slowed speech input $(t(21)=2.54, p=0.019)$.

\section{Discussion}

The results reveal that the interpretation of pronouns improves from chance performance at a regular rate of speech input to better-than-chance performance with slowed speech input. In contrast, the interpretation of reflexives is quite good with regular rate input, and does not improve (or get worse) with slowed speech input.

As with the on-line results, we examined the potential of age effects in task performance. We correlated age with accuracy for pronouns and accuracy for reflexives for each rate of speech for each group. At a regular rate of speech, there was a strong correlation between age and pronoun accuracy $(r=0.656, p=0.001)$, and an interesting pattern emergedthere appears to be a discontinuous distribution of scores (Fig. 5), with children above age 8 performing almost perfectly, and children below age 8 performing at or below chance. While there was a nearly significant correlation between age and reflexive performance as well ( $r=0.384, p=0.086)$, there was no discontinuity in the distribution of scores. This is consistent with the observation that pronoun interpretation may be slower to develop than reflexive interpretation, and may not have developed before age 8 in TLD children.

At slowed rates of speech input (Fig. 5), there was again a correlation between age and pronoun accuracy ( $r=0.452, p=0.035)$, but with a more continuous distribution, such that it appears that the younger children performed better (fewer subjects were below chance). The correlation between age and reflexive performance also reached significance with slowed speech $(r=0.428, p=0.047)$, suggesting that some children can benefit from slowed input on reflexives and therefore may not have fully mastered off-line processing of these forms. 

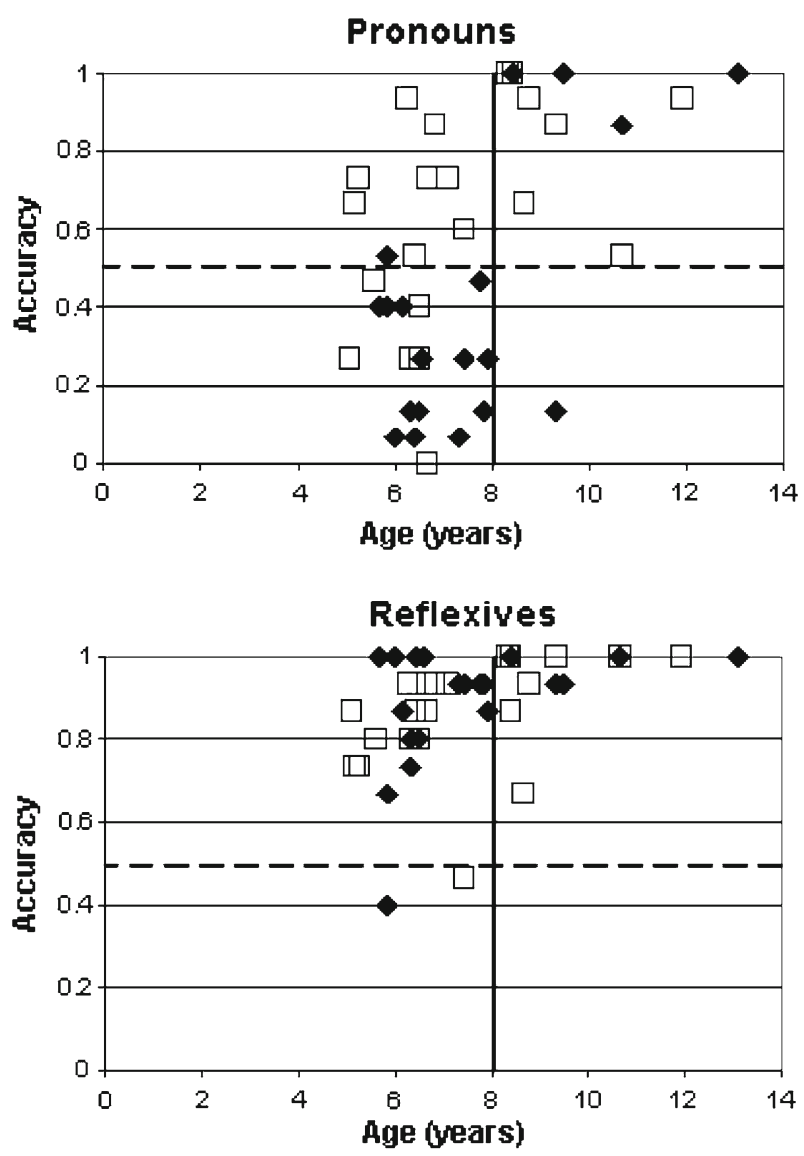

Fig. 5 A plot of age versus accuracy for pronouns and reflexives at regular (solid diamond) and slowed (open square) rates of speech input. The dashed horizontal line indicates chance performance (50\% accuracy). The thick vertical line separates children above and below 8 years old. Note that 43 participants' data are represented on this graph, however, symbols with the same or similar values may be overlapping and thus not easily distinguishable

\section{Conclusions}

In sum, at a regular rate of speech input, young children (aged 5-13) demonstrated correct activation of the antecedent of a pronoun in an on-line priming paradigm. However, the same children showed impaired (chance level) off-line comprehension of pronouns with substantially better comprehension of reflexives.

In addition, age effects were found for both on-line and off-line studies. On-line, older children responded more quickly than younger children, though there was no difference in this age effect across conditions (i.e., with age treated as a continuous variable)—suggesting that children of different ages (within our age range of 5-13) were not performing differently in the processing of these constructions. Off-line, children's comprehension of pronouns was at or below chance levels until about age 8 - children older than 8 showed vastly better comprehension of these forms. This age cut-off is very close to prior findings that children younger than 7 show particular deficits in pronominal dependency relationships (van der 
Lely and Stollwerck 1997). In contrast, children as young as 6 years of age showed competent performance on reflexives in our off-line study, though performance did improve with increased age. However, unlike for the pronouns, there was no clear break in performance levels across the age range we tested, with even the youngest children performing at higher than chance levels (except in one case).

This pattern of results for pronouns-adult-like on-line performance and developmentally delayed off-line performance-is consistent with prior studies of on-line and off-line pronoun comprehension in young children (see Introduction). That is, the results obtained here support the claim that children as young as 4 or 5 have fully developed (i.e., adultlike) automatic structural processing routines (Love 2007; McKee et al. 1993; Roberts et al. 2007), whereas their meta-linguistic knowledge of pronoun-antecedent relations (necessary for off-line task performance), is under-developed. This dissociation in performance also underscores the need to examine child language processing from the perspective of multiple methods.

The effects of slowed speech input were clearly different for the on-line and off-line studies. On-line, slowing the rate of speech input to an abnormally slow rate impaired performance-children did not prime the antecedent of the pronoun. Moreover, it appeared that children were incorrectly priming the non-local main clause subject as an antecedent for the reflexive-suggesting that slowed speech input adversely affects automatic structural processing for both pronouns (failing to prime the correct antecedent) and reflexives (priming a structurally invalid antecedent). In contrast, a slowed rate of speech input led to better-thanchance performance for pronoun comprehension in the off-line study (but did not appear to have any impact on reflexive performance).

These slow rate results raise the question: Why does slowed-speech input have a differential effect on on-line and off-line task performance? Specifically, for pronouns, why does slow-rate speech lead to impaired performance on-line, but improved performance off-line? Similarly, why does slow-rate speech lead to abnormal on-line priming for reflexives, but no change in off-line comprehension of reflexives?

It appears that typical-language developing children have mastered the real time language processing skills needed for long distance dependency relationships (including pronouns and reflexives). This is supported by findings (here and in prior studies) of adult-like patterns of priming for co-reference processing at regular speech rates, for pronouns and other co-referential structural elements (see above). Given this mastery, the process is fast-acting and automatic, but breaks down (for both pronouns and reflexives) when the speech input rate is outside the "normal" speech rate range for which the process is optimized-consistent with previous claims of adult-language processing (Love et al. 2008).

In contrast, as children's off-line performance for pronouns appears to be developmentally delayed, they may not yet have achieved sufficient mastery to rely on automatic processes, and are instead dependent on meta-linguistic, reflective processes. We posit that a slowed rate of speech input leads to improved performance on these slower, reflective processes due to the additional time afforded the parser, which aids the child in the off-line decision making process. For reflexives, a slowed rate of speech input did not lead to improved off-line performance. If anything, the slowed input may have slightly impaired performance on average, though this was at best a miniscule effect (and not statistically reliable). One possibility is that the children may have been at ceiling already, as they performed above $85 \%$ at the regular rate of speech. However, if they were at ceiling, why shouldn't slowed input have disrupted performance, as found previously for adults (Love et al. 2008)? While there is some slight indication of impaired performance, this level of performance $(85 \%)$ may be ceiling performance for children of this age range, but it may not yet be good enough to indicate a 
reliance on automatic rather than meta-linguistic processes-contrast off-line performance of adults, who remained above $95 \%$ accuracy on off-line co-reference processing, even when impaired with slowed input (Love et al. 2008).

In summary, we suggest that the slowed speed of the incoming auditory speech helped aspects of language processing that are not fully developed in children (off-line pronoun performance) while impairing those aspects of language processing that they have mastered (on-line co-reference processing). Future research investigating the impact of slowed-speech on language impaired children from this perspective may lead to the identification and development of possible parameters for treatment studies.

Acknowledgments The authors wish to thank Maxwell Moholy, Brianne Bricker, Justin Baker, Danielle Vignati, Richard Schwartz and Arild Hestvik for their contributions at various stages of this project. This work was supported by the National Institutes of Health, R01DC03885.

Open Access This article is distributed under the terms of the Creative Commons Attribution Noncommercial License which permits any noncommercial use, distribution, and reproduction in any medium, provided the original author(s) and source are credited.

\section{References}

Baauw, S., \& Cuetos, F. (2003). The interpretation of pronouns in Spanish language acquisition and breakdown: Evidence for the "Principle B Delay" as a non-unitary phenomenon. Language Acquisition: A Journal of Developmental Linguistics, 11(4), 219-275. doi:10.1207/s15327817la1104_2.

Baayen, R. H. (2004). Statistics in psycholinguistics: A critique of some current gold standards. Mental Lexicon Working Papers 1, 1, 1-47.

Baayen, R. H. (2008). Analyzing linguistic data: A practical introduction to statistics. New York: Cambridge University Press.

Balogh, J., Zurif, E., Prather, P., Swinney, D., \& Finkel, L. (1998). Gap-filling and end-of-sentence effects in real-time language processing: Implications for modeling sentence comprehension in aphasia. Brain and Language, 61(2), 169-182. doi:10.1006/brln.1997.1917.

Booth, J. R., MacWhinney, B., \& Harasaki, Y. (2000). Developmental differences in visual and auditory processing of complex sentences. Child Development, 71(4), 981-1003. doi:10.1111/1467-8624.00203.

Brown, L., Sherbenou, R. J., \& Johnsen, S. K. (1997). Test of nonverbal intelligence (3rd ed). Austin, TX: PRO-ED.

Camblin, C. C., Ledoux, K., Boudewyn, M., Gordon, P. C., \& Swaab, T. Y. (2007). Processing new and repeated names: Effects of coreference on repetition priming with speech and fast RSVP. Brain Research, 1146, 172-184. doi:10.1016/j.brainres.2006.07.033.

Chien, Y.-C., \& Wexler, K. (1990). Children's knowledge of locality conditions in binding as evidence for the modularity of syntax and pragmatics. Language Acquisition: A Journal of Developmental Linguistics, 1(3), 225-295. doi:10.1207/s153278171a0103_2.

Chomsky, N. (1981). Lectures on government and binding. Dordrecht: Foris.

Chomsky, N. (1986). Knowledge of language: Its nature, origin and use. New York: Praeger.

Cowles, H. W., Walenski, M., \& Kluender, R. (2007). Linguistic and cognitive prominence in anaphor resolution: topic, contrastive focus and pronouns. Topoi, 26(1), 3-18. doi:10.1007/s11245-006-9004-6.

Gardner, H., Albert, M., \& Weintraub, S. (1975). Comprehending a word: The influence of speed and redundancy on auditory comprehension in aphasia. Cortex, 11, 155-162.

Grimshaw, J. R. S. (1990). Knowledge and obedience: The developmental status of the binding theory. Linguistic Inquiry, 21, 187-222.

Grodzinsky, Y., \& Kave, G. (1994). Do children really know condition A? Language Acquisition: A Journal of Developmental Linguistics, 3(1), 41-45. doi:10.1207/s153278171a0301_2.

Grodzinsky, Y., Wexler, K., Chien, Y., Marakovitz, S., \& Solomon, J. (1993). The breakdown of binding relations. Brain and Language, 45, 396-422. doi:10.1006/brln.1993.1052.

Hestvik, A., Schwartz, R., Tornyova, L., Almodovar, D., Love, T., \& Swinney, D. (2007). Binding theory and syntactic movement are dissociated in specific language impairment. Paper presented at the Twentieth Annuam CUNY Conference on Human Sentence Processing, La Jolla, CA. 
Kail, R., \& Hall, L. K. (1994). Processing speed, naming speed, and reading. Developmental Psychology, 30(6), 949-954. doi:10.1037/0012-1649.30.6.949.

Koster, C. (1993). Errors in anaphora acquisition. Unpublished Doctoral Dissertation, Utrecht University.

Lasky, E. Z., Weidner, W. E., \& Johnson, J. P. (1976). Influence of linguistic complexity, rate of presentation, and interphase pause time on auditory-Verbal comprehension of adult aphasic patients. Brain and Language, 3, 386-395. doi:10.1016/0093-934X(76)90034-1.

Love, T. (2007). The processing of non-canonically ordered constituents in long distance dependencies by pre-school children: A real-time investigation. Journal of Psycholinguistic Research, 36, 191-206. doi:10. 1007/s10936-006-9040-9.

Love, T., \& Swinney, D. (1996). Coreference processing and levels of analysis in object-relative constructions: Demonstration of antecedent reactivation with the cross-modal priming paradigm. Journal of Psycholinguistic Research, 25, 5-24. doi:10.1007/BF01708418.

Love, T., Swinney, D., Walenski, M., \& Zurif, E. (2008). How left inferior frontal cortex participates in syntactic processing: Evidence from aphasia. Brain and Language, 107(3), 203-219.

McDaniel, D., Cairns, H. S., \& Hsu, J. R. (1990). Binding principles in the grammars of young children. Language Acquisition: A Journal of Developmental Linguistics, 1(1), 121-138. doi:10.1207/ s15327817la0101_5.

McDaniel, D., \& Maxfield, T. L. (1992). Principle B and contrastive stress. Language Acquisition: A Journal of Developmental Linguistics. Special Issue: The Development of Binding, 2(4), 337-358.

McKee, C. (1992). A comparison of pronouns and anaphors in Italian and English acquisition. Language Acquisition: A Journal of Developmental Linguistics, 2, 21-54. doi:10.1207/s15327817la0201_2.

McKee, C., Nicol, J., \& McDaniel, D. (1993). Children's application of binding during sentence processing. Language and Cognitive Processes, 8(3), 265-290. doi:10.1080/01690969308406956.

Nicol, J. L. (1988). Coreference processing during sentence comprehension. Ph.D. Thesis, Massachusetts Institute of Technology, Cambridge, MA.

Nicol, J., Fodor, J. D., \& Swinney, D. (1994). Using cross-modal lexical decision tasks to investigate sentence processing. Journal of Experimental Psychology. Learning, Memory, and Cognition, 20(5), 1229-1238. doi:10.1037/0278-7393.20.5.1229.

Nicol, J., \& Swinney, D. (1989). The role of structure in coreference assignment during sentence comprehension. Journal of Psycholinguistic Research, 18, 5-19. doi:10.1007/BF01069043.

Nicol, J., \& Swinney, D. (2002). The psycholinguistics of anaphora. Boston: Blackwell Publishing.

Nicol, J., Swinney, D., Love, T., \& Hald, L. (2006). The on-line study of sentence comprehension: An examination of dual task paradigms. Journal of Psycholinguistic Research, 35(3), 215-231. doi:10.1007/ s10936-006-9012-0.

Pashek, G. V., \& Brookshire, R. H. (1982). Effects of rate of speech and linguistic stress on auditory paragraph comprehension of aphasic individuals. Journal of Speech and Hearing Research, 25, 377-383.

Poeck, K., \& Pietron, H.-P. (1981). The influence of stretched speech on token test performance of aphasic and right-brain damaged patients. Neuropsychologia, 19, 133-136. doi:10.1016/0028-3932(81)90052-X.

Radeau, M., Morais, J., Mousty, P., \& Bertelson, P. (2000). The Effect of speaking rate on the role of the uniqueness point in spoken word recognition. Journal of Memory and Language, 42, 406-422. doi:10. 1006/jmla.1999.2682.

Roberts, L., Marinis, T., Felser, C., \& Clahsen, H. (2007). Antecedent priming at trace positions in children's sentence processing. Journal of Psycholinguistic Research, 36, 175-188. doi:10.1007/s10936-006-9038-3.

Sag, I., \& Wasow, T.(1999). Syntactic theory: A formal introduction. Stanford: CSLI.

Satterthwaite, F. E. (1946). An approximate distribution of estimates of variance components. Biometrics Bulletin, 2(6), 110-114. doi:10.2307/3002019.

Sekerina, I. A., Stromswold, K., \& Hestvik, A. (2004). How do adults and children process referentially ambiguous pronouns? Journal of Child Language, 31(1), 123-152. doi:10.1017/S0305000903005890.

Semel, E., Wiig, E. H., \& Secord, W. A. (2003). Clinical evaluation of language fundamentals (4th ed.) San Antonio, TX: The Psychological Corporation.

Solan, L. (1983). Pronominal reference: Child language and the theory of grammar. Dordrecht, Holland: D. Reidel Publishing Company.

Solan, L., \& Ortiz, R. (1982). The development of pronouns and reflexives: Evidence from Spanish. Paper presented at the Sixth Boston University Conference on Child Language Development, Boston, MA.

Swinney, D. (1979). Lexical access during sentence comprehension: (Re)consideration of context effects. Journal of Verbal Learning and Verbal Behavior, 5, 219-227.

Swinney, D., \& Love, T.(1998). Language input as a parameter on structural processing. Paper presented at the Eleventh Annual CUNY Conference on Human Sentence Processing. New Brunswick, NJ.

Swinney, D., Onifer, W., Prather, P., \& Hirshkowitz, M. (1979). Semantic facilitation across sensory modalities in the processing of individual words and sentences. Memory \& Cognition, 7, 159-165. 
Swinney, D., \& Prather, P. (1989). On the comprehension of lexical ambiguity by young children: Investigations into the development of mental modularity. In D. Gorfein (Ed.), Resolving semantic ambiguity (pp. 225-238). New York: Springer.

Swinney, D., Zurif, E., Prather, P., \& Love, T. (1996). Neurological distribution of processing operations underlying language comprehension. Journal of Cognitive Neuroscience, 8(2), 174-184. doi:10.1162/jocn.1996. 8.2.174.

Tallal, P., \& Piercy, M. (1973a). Defects of nonverbal auditory perception in children with developmental dysphasia. Nature, 241, 468-469. doi:10.1038/241468a0.

Tallal, P., \& Piercy, M. (1973b). Developmental aphasia: Impaired rate of non-verbal processing as a function of sensory modality. Neuropsychologia, 11, 389-398. doi:10.1016/0028-3932(73)90025-0.

Tallal, P., \& Piercy, M. (1974). Developmental aphasia: Rate of auditory processing as a selective impairment of consonant perception. Neuropsychologia, 12, 83-93. doi:10.1016/0028-3932(74)90030-X.

Tallal, P., \& Piercy, M. (1975). Developmental aphasia: The perception of brief vowels and extended stop consonants. Neuropsychologia, 13(1), 69-74. doi:10.1016/0028-3932(75)90049-4.

Tauroza, S., \& Allison, D. (1990). Speech rates in British English. Applied Linguistics, 11(1), 90-105. doi:10. 1093/applin/11.1.90.

van der Lely, H. K. J., \& Stollwerck, L. (1997). Binding theory and grammatical specific language impairment in children. Cognition, 62, 245-290. doi:10.1016/S0010-0277(96)00783-4.

van Heuven, V. J., \& van Zanten, E. (2005). Speech rate as a secondary prosodic characteristic of polarity questions in three languages. Speech Communication. Special Issue In Honour of Louis Pols, 47(1-2), 87-99.

Walenski, M. (2002). Relating parsers and grammars: On the structure and real-time comprehension of English infinitival complements. Unpublished Doctoral Dissertation, University of California San Diego, San Diego.

Walenski, M., Mostofsky, S. H., \& Ullman, M. T. (2007). Speeded processing of grammar and tool knowledge in Tourette's syndrome. Neuropsychologia, 45, 2447-2460. doi:10.1016/j.neuropsychologia.2007.04.001.

Ziegler, W. (2002). Task-related factors in oral motor control: Speech and oral diadochokinesis in dysarthria and apraxia of speech. Brain and Language, 80, 556-575. doi:10.1006/brln.2001.2614. 